Pacific Journal of Mathematics

DERIVATIONS OF HIGHER ORDER AND COMMUTATIVITY
OF RINGS 


\title{
DERIVATIONS OF HIGHER ORDER AND COMMUTATIVITY OF RINGS
}

\author{
L. O. Chung AND JiAng LUH
}

Let $R$ be an associative ring with center $C$, and $\partial$ be a derivation on $R$. The authors consider the commutativity of $R$ which satisfies the property that $\partial^{n} x-\partial^{m} x \in C$, where $n>m$ are fixed nonnegative integers. An example is given to show that if $m \geqq 2, R$ may not be commutative. For $0 \leqq m \leqq 2$, suppose $R$ is either $r$-torsion free with large $r$ or torsion free. It is shown that (i) if $\partial^{n} x \pm x \in C$ for all $x \in R$ then all commutators of $R$ are central; (ii) if $\partial^{n} x \pm$ $\partial x \in C$ for all $x \in R$ and $n$ is even then $\partial x \partial y-\partial y \partial x \in C$ for all $x, y \in R$; (iii) if $\partial^{n} x \pm \partial^{2} x \in C$ for all $x \in R$ and if $n$ is odd then $\partial^{2} x \partial^{2} y-\partial^{2} y \partial^{2} x \in C$ for all $x, y \in R$. In all these cases, if one assumes further that $R$ is prime, then $\partial$ must be trivial. Examples are also given to illustrate that some of these assumptions on evenness of $n$, and that $r$ being large are essential. Finally, those integral domains which have $\partial^{n} x$ central for all $x$ are also studied. They are shown to be commutative.

Wedderburn's theorem, asserting that a finite division ring is necessarily commutative, has been generalized in several directions [2]. A well-known theorem of Jacobson states that if, for each $x$ in a ring $R$, there exists an integer $n>1$, depending upon $x$, such that $x^{n}=x$, then $R$ is commutative. Herstein generalizes this result further. He proves that, if for each $x$ in a ring $R$ there exists an integer $n>1$, depending upon $x$, such that $x^{n}-x$ is central, then $R$ is commutative. Let us now examine some aspects of finite division rings. Let $R$ be a finite division ring of order $p^{k}$, where $p$ is a prime. For $a \in R$, let $\partial: x \mapsto a x-x a$ for all $x \in R$. It can be easily seen that $\partial^{p^{k}} x=\partial x$ for all $x \in R$. From this observation, a natural question arises: Let $R$ be a ring and $\partial$ be a nontrivial derivation on $R$. If, for all $x \in R, \partial^{n} x-\partial^{m} x$ is central, where $n>m$ are fixed nonnegative integers, does this force $R$ to be commutative or almost so? An example is given to show that the answer to the question is negative if $m>2$. For $0 \leqq m \leqq 2$, we shall show: Suppose $R$ is a ring with center $C$ which is either $r$-torsion free with large $r$ or torsion free. (i) If $\partial^{n} x \pm x \in C$ for all $x \in R$ then $x y-y x \in C$ for all $x, y \in R$; (ii) if $\partial^{n} x \pm \partial x \in C$ for all $x \in R$ and if $n$ is even then $\partial x \partial y-\partial y \partial x \in C$ for all $x, y \in R$; (iii) if $\partial^{n} x \pm \partial^{2} x \in C$ for all $x \in R$ and if $n$ is odd then $\partial^{2} x \partial^{2} y-\partial^{2} y \partial^{2} x \in C$ for all $x, y \in R$. In all these cases, if we assume $R$ is prime then $\partial$ must be trivial. Examples are also 
given to illustrate that some of these assumptions on evenness of $n$, and that $r$ being large are essential. Finally those integral domains which have $\partial^{n} x$ central for all $x$ are also studied. They are shown to be commutative.

In the proofs of these main results, we use certain circulant determinants of binomial coefficients which have been recognized to have close connections with Fermat's Last Theorem. Many problems concerning these determinants are still open. Without answers to these problems, it does not seem to be easy to improve our main results by weakening the hypothesis on the characteristic of rings without a completely different approach.

1. Preliminaries. Throughout this paper $R$ denotes an associative ring with center $C, Z$ denotes the ring of integers and $Z^{+}$the set of positive integers. A derivation $\partial$ on $R$ is a mapping of $R$ into itself such that $\partial(x+y)=\partial x+\partial y$ and $\partial(x y)=x \partial y+\partial x y$ for all $x, y \in R$. For $x, y \in R,[x, y]$ denotes the commutator $x y-y x$. For $n \in Z^{+}$, we denote by $D_{n}$ the following matrix of binomial coefficients:

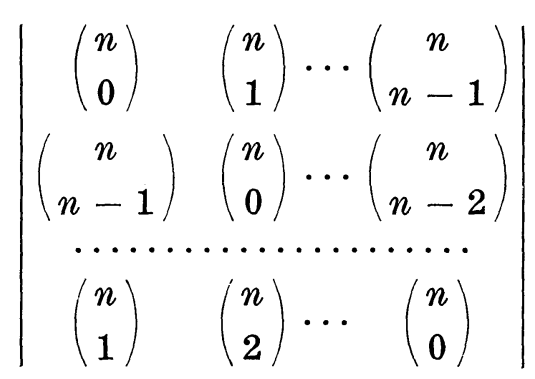

and by $\Delta_{n}$ the determinant of $D_{n}$. It is well-known that $\Delta_{n}=0$ if and only if $6 \mid n$. Moreover, $\Delta_{n}=\prod_{j=1}^{n}\left(\left(1+\xi^{j}\right)^{n}-1\right)$, where $\xi$ is an $n$th primitive root of unity. Little is known about the prime factors of $\Delta_{n}$. Some close connections between $\Delta_{n}$ and Fermat's Last Theorem have been cited in several papers [1, 4].

The following proposition will be useful in the sequel.

Proposition 1.1. Let

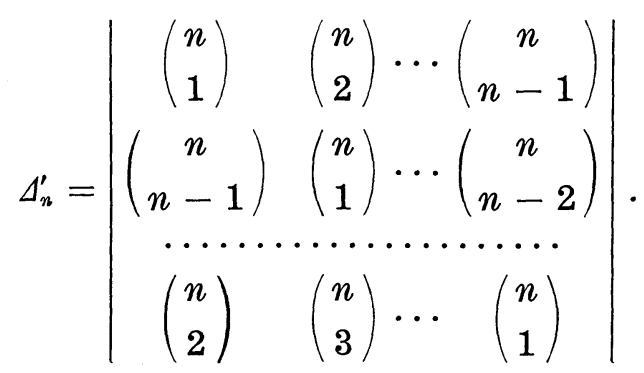

Then $\Delta_{n}^{\prime}=0$ if $n$ is odd and $\Delta_{n}^{\prime}=2 \Delta_{n-1}$ if $n$ is even. 
Proof. Replacing $\left(\begin{array}{l}n \\ k\end{array}\right)$ by $\left(\begin{array}{l}n-1 \\ k-1\end{array}\right)+\left(\begin{array}{c}n-1 \\ k\end{array}\right)$ in each entry of the determinant $\Delta_{n}^{\prime}$, we can easily see that $\Delta_{n}^{\prime}=\Delta_{n-1}+(-1)^{n-2} \Delta_{n-1}=$ $\left(1+(-1)^{n-2}\right) \Delta_{n-1}$.

\section{General rings. We begin with}

THEOREM 2.1. If $\partial^{n} x-\partial^{2} x \in C$ for all $x \in R$, where $n$ is an odd integer $>2$ and if $R$ is $2 \Delta_{n-2}$-torsion free, then $\left[\partial^{2} x, \partial^{2} y\right] \in C$.

Proof. Let $x, y \in R . \quad \partial^{n}[x, y]-\partial^{2}[x, y] \in C$ yields

$$
\sum_{j=1}^{n-1}\left(\begin{array}{l}
n \\
j
\end{array}\right)\left[\partial^{n-j} x, \partial^{j} y\right]-2[\partial x, \partial y] \in C
$$

In (2.1), replacing $x$ by $\partial x$ and $y$ by $\partial y$ and using $\left[\partial^{n} x, \partial^{2} y\right]=$ $\left[\partial^{2} x, \partial^{2} y\right]=\left[\partial^{2} x, \partial^{n} y\right]$, we obtain $(2 n-2)\left[\partial^{2} x, \partial^{2} y\right]+\sum_{j=2}^{n-2}\left(\begin{array}{c}n \\ j\end{array}\right)\left[\partial^{n-j+1} x\right.$, $\left.\partial^{j+1} y\right] \in C$, or

$$
\sum_{j=1}^{n-2} a_{j}\left[\partial^{n-j+1} x, \partial^{j+1} y\right] \in C
$$

where $a_{1}=2(n-1), a_{j}=\left(\begin{array}{c}n \\ j\end{array}\right)$ for $j \geqq 2$.

In (2.2), we replace $x$ by $\partial^{n-2-i} x, y$ by $\partial^{i} y, i=0,1,2, \cdots, n-3$. We obtain $n-2$ forms of central elements which can be expressed by the following matrix form:

$$
\left(\begin{array}{cccc}
a_{1} & a_{2} & \cdots & a_{n-2} \\
a_{n-2} & a_{1} & \cdots & a_{n-3} \\
\cdots & \cdots & \cdots & \cdots \\
a_{2} & a_{3} & \cdots & a_{1}
\end{array}\right)\left(\begin{array}{c}
{\left[\partial^{2} x, \partial^{2} y\right]} \\
{\left[\partial^{n-1} x, \partial^{3} y\right]} \\
{\left[\partial^{n-2}, \partial^{4} y\right]} \\
\vdots \\
{\left[\partial^{3} x, \partial^{n-1} y\right]}
\end{array}\right)=\left(\begin{array}{c}
c_{1} \\
c_{2} \\
\vdots \\
c_{n-2}
\end{array}\right)
$$

where all $c_{i} \in C$.

Note that $a_{1}=\left(\begin{array}{l}n-1 \\ n-2\end{array}\right)+\left(\begin{array}{c}n-1 \\ 1\end{array}\right)$ and $a_{j}=\left(\begin{array}{c}n-1 \\ j-1\end{array}\right)+\left(\begin{array}{c}n-1 \\ j\end{array}\right)$ for $j \geqq 2$. Thus

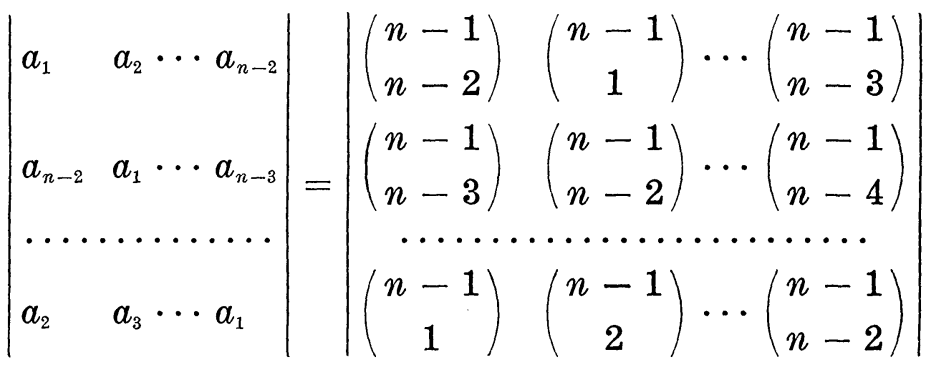




$$
\begin{aligned}
& +\left|\begin{array}{ccc}
\left(\begin{array}{c}
n-1 \\
1
\end{array}\right) & \left(\begin{array}{c}
n-1 \\
2
\end{array}\right) \cdots\left(\begin{array}{c}
n-1 \\
n-2
\end{array}\right) \\
\left(\begin{array}{c}
n-1 \\
n-2
\end{array}\right) & \left(\begin{array}{c}
n-1 \\
1
\end{array}\right) \cdots\left(\begin{array}{c}
n-1 \\
n-3
\end{array}\right) \\
\ldots \ldots \ldots & \ldots \ldots \ldots \ldots\left(\begin{array}{c}
n-1 \\
1
\end{array}\right)
\end{array}\right| \\
& =(-1)^{n-3} \Delta_{n-1}^{\prime}+\Delta_{n-1}^{\prime}=2 \Delta_{n-1}^{\prime}=4 \Delta_{n-2}
\end{aligned}
$$

by Proposition 1.1. Now, premultiplication of the adjoint of the "circulant" matrix in both sides of (2.3) yields

$$
2^{2} \Delta_{n-2}\left(\begin{array}{l}
{\left[\partial^{2} x, \partial^{2} y\right]} \\
{\left[\partial^{n-1} x, \partial^{3} y\right]} \\
\vdots \\
{\left[\partial^{3} x, \partial^{n-1} y\right]}
\end{array}\right)=\left(\begin{array}{c}
c_{1}^{\prime} \\
c_{2}^{\prime} \\
\vdots \\
c_{n-2}^{\prime}
\end{array}\right),
$$

where all $c_{i}^{\prime} \in C$. Consequently, $2^{2} \Delta_{n-2}\left[\partial^{2} x, \partial^{2} y\right]=c_{1}^{\prime} \in C$. Since $R$ is $2 \Delta_{n-2}$-torsion free, it follows that $\left[\partial^{2} x, \partial^{2} y\right] \in C$ as desired.

COROLLARY. If $R$ is also $(n-2)$-torsion free then $[\partial x, \partial y] \in C$ for all $x, y \in R$.

Proof. According to Theorem 2.1, $\left[\partial^{h} x, \partial^{k} y\right] \in C$ for all $x, y \in R$ if $h, k \geqq 2$. Now, in (2.1), we replace $x$ by $\partial x$. It follows that $(n-2)\left[\partial^{2} x, \partial y\right] \in C$. Since $R$ is $(n-2)$-torsion free, $\left[\partial^{2} x, \partial y\right] \in C$. Likewise $\left[\partial x, \partial^{2} y\right] \in C$. Thus, from (2.1), $[\partial x, \partial y] \in C$.

We should note that although the condition, " $2(n-2) \Delta_{n-2}$-torsion free" could be weakened in the corollary, the condition that $R$ being 2-torsion free is essential. This can be seen from the following.

EXAMPLE. Let $A$ be the $3 \times 3$ matrix ring over $G F(2)$, the Galois field of order 2 , and $\partial$ be the inner derivation:

$$
\partial x=\left[\left(\begin{array}{lll}
0 & 0 & 1 \\
0 & 0 & 0 \\
0 & 0 & 0
\end{array}\right), x\right] \quad \text { for } x \in A
$$

Then $\partial^{2}=0$ and $\partial^{n} x-\partial^{2} x \in C$, the center of $A$ for $n>2$. However $[\partial x, \partial y]$ need not be in the center of $A$.

THEOREM 2.2. If $\partial^{n} x-\partial x \in C$ for all $x \in R$, where $n$ is an even 
integer $>1$ and if $R$ is $2 \Delta_{n-1}$-torsion free, then $[\partial x, \partial y] \in C$ for all $x, y \in R$.

Proof. Noting that $\partial$ maps $C$ into itself, $\partial^{n} x-\partial x \in C$ implies $\partial^{n+1} x-\partial^{2} x \in C$ which by Theorem 2.1 implies $\left[\partial^{2} x, \partial^{2} y\right] \in C$ for all $x$, $y \in R$. Thus, $\left[\partial x, \partial^{2} y\right]=\left[\partial^{n} x, \partial^{2} y\right] \in C$. Since $\left[\partial^{n} x-\partial x, \partial^{n} y-\partial y\right]=0$, it follows that $[\partial x, \partial y]=\left[\partial x, \partial^{n} y\right]+\left[\partial^{n} x, \partial y\right]-\left[\partial^{n} x, \partial^{n} y\right] \in C$ as we desired.

The assumption in Theorem 2.2 that $n$ is even is essential.

ExAmple. Let $A$ be the $2 \times 2$ matrix ring over a field $F$ of characteristic $\neq 2$ and $\partial$ be the inner derivation:

$$
\partial\left(\begin{array}{ll}
x & y \\
z & u
\end{array}\right)=\left[\left(\begin{array}{ll}
1 & 0 \\
0 & 0
\end{array}\right),\left(\begin{array}{ll}
x & y \\
z & u
\end{array}\right)\right]=\left(\begin{array}{rr}
0 & y \\
-z & 0
\end{array}\right) .
$$

Then, for any odd integer $n, \partial^{n}\left(\begin{array}{ll}x & y \\ z & u\end{array}\right)-\partial\left(\begin{array}{ll}x & y \\ z & u\end{array}\right)=\left(\begin{array}{ll}0 & 0 \\ 0 & 0\end{array}\right)$. However $[\partial X, \partial Y]$ need not be central.

Now we consider those rings having derivation $\partial$ which satisfy $\partial^{n} x-x \in C$ for all $x \in R$. Clearly, in this case, $\partial^{n+1} x-\partial x \in C$ for all $x \in R$. Thus, if $R$ is $2 \Delta_{n}$-torsion free and if $n$ is odd, by Theorem $2.2[\partial x, \partial y] \in C$ for all $x, y \in R$. In fact we are able to show that $[x, y] \in C$ for all $x, y \in R$ even without assuming that $n$ is odd.

THEOREM 2.3. If $\partial^{n} x-x \in C$ for all $x \in R$ and if $R$ is $\Delta_{n}$-torsion free, then $[x, y] \in C$ for all $x, y \in R$.

Proof. For $x, y \in R, \partial^{n}[x, y]-[x, y] \in C$ yields

$$
\sum_{j=1}^{n}\left(\begin{array}{c}
n \\
j
\end{array}\right)\left[\partial^{n-j} x, \partial^{j} y\right] \in C \text {. }
$$

We replace $x$ by $\partial^{i} x$ and $y$ by $\partial^{n-i} y$, where $i=1,2, \cdots, n$. We obtain

$$
\sum_{j=1}^{n}\left(\begin{array}{c}
n \\
j
\end{array}\right)\left[\partial^{n-j+i} x, \partial^{n-i+j} y\right] \in C
$$

By noting that $\partial^{m} z-\partial^{t} z \in C$ for all $z \in R$ provided $m \equiv t(\bmod n)$, we can see easily that, for each $i \in Z^{+}$with $i \leqq n$, the set $\{n-1+i$, $n-2+i, \cdots, n-n+i\}$ is a complete residue system modulo $n$. Thus, after rearranging the terms in the left hand side of (2.5) for $i=0, n-1, n-2, \cdots, 1$, we can express the $n$ forms of central elements by the following matrix form: 


$$
D_{n}\left(\begin{array}{l}
{\left[x, \partial^{n} y\right]} \\
{\left[\partial^{n-1} x, \partial y\right]} \\
\vdots \\
{\left[\partial x, \partial^{n-1} y\right]}
\end{array}\right)=\left(\begin{array}{c}
c_{1} \\
c_{2} \\
\vdots \\
c_{n}
\end{array}\right),
$$

where $c_{1}, c_{2}, \cdots, c_{n} \in C$ and $D_{n}$ is the matrix defined in $\S 1$.

Premultiplying by the adjoint of $D_{n}$, we get

$$
\Delta_{n}\left(\begin{array}{l}
{\left[x, \partial^{n} y\right]} \\
{\left[\partial^{n-1} x, \partial y\right]} \\
\vdots \\
{\left[\partial x, \partial^{n-1} y\right]}
\end{array}\right)=\left(\begin{array}{c}
c_{1}^{\prime} \\
c_{2}^{\prime} \\
\vdots \\
c_{n}^{\prime}
\end{array}\right),
$$

where $c_{1}^{\prime}, c_{2}^{\prime}, \cdots, c_{n}^{\prime} \in C$ and $\Delta_{n}$ is the determinant of $D_{n}$. Particularly, $\Delta_{n}[x, y]=\Delta_{n}\left[x, \partial^{n} y\right]=c_{n}^{\prime} \in C$. Since, by our assumption, $R$ is $\Delta_{n^{-}}$ torsion free, we obtain $[x, y] \in C$ as desired.

3. Prime rings. In this section we shall study prime ring $R$ with nontrivial derivation $\partial$ which satisfies $\partial^{n} x-\partial^{m} x \in C$.

LEMMa 3.1. Let $R$ be a prime ring with nontrivial derivation o. If $\partial x \in C$ for all $x \in R$ then $R$ is commutative.

Proof. For $x, y \in R,[\partial(x y), y]=0$ yields $0=[\partial x y, y]+[x \partial y, y]=$ $\partial y[x, y]$. So either $\partial y=0$ or $[x, y]=0$. Let $K=\{y \in R \mid \partial y=0\}$. Then $K$ is an additive proper subgroup of $R$ and $K \cup C=R$. A well-known theorem in group theory states that a group cannot be a union of two proper subgroups. Thus $C=R$ and $R$ is commutative.

In [3], Herstein proves that, for a prime ring $R$ of characteristic $\neq 2$ with nontrivial derivation $\partial$, if $[\partial x, \partial y]=0$ for all $x, y \in R$ then $R$ is necessarily commutative. Here we shall show that $[\partial x, \partial y]=0$ for all $x, y \in R$ is equivalent to the condition that $\partial^{2} x \in C$ for all $x \in R$. Using this fact we shall show that $R$ is commutative. Thus, this will provide another proof of the result of Herstein.

THEOREm 3.2. If $R$ is prime of characteristic $\neq 2$ and $\partial$ is a nontrivial derivation on $R$, then the following are equivalent:

(i) $\partial^{2} x \in C$ for all $x \in R$;

(ii) $[\partial x, \partial y]=0$ for all $x, y \in R$;

(iii) $R$ is commutative.

Proof. We should note that $\partial^{2} R=0$ forces $\partial R=0$.

(i) $\Rightarrow$ (ii): Commute $\partial^{2}(x \partial y) \in C$ with $x$ to obtain $0=\partial^{2} x[\partial y, x]+$ 
$2 \partial y\left[\partial^{2} x, x\right]$. Replace $x$ with $\partial x$ and use the subgroup argument of Lemma 3.1 to get either (ii) or $\partial^{3} R=0$. If $\partial^{3} R=0$, commuting $\partial^{2}(x \partial y) \in C$ with $\partial x$ gives $\partial^{2} x[\partial y, \partial x]=0$. Again (ii) holds or $\partial^{2} R=0$ forcing $\partial R=0$.

(ii) $\Rightarrow$ (iii): This is a Herstein's result [3]. For easy reference, we exhibit a proof here. Assume (ii). For $x, y, z \in R, 0=$ $[\partial(\partial x y), \partial z]=\left[\partial^{2} x y+\partial x \partial y, \partial z\right]=\left[\partial^{2} x y, \partial z\right]=\partial^{2} x[y, \partial z]$. By replacing $y$ by $y w$, we obtain $0=\partial^{2} x[y w, \partial z]=\partial^{2} x(y w \partial z-\partial z y w)=\partial^{2} x y[w, \partial z]+$ $\partial^{2} x[y, \partial z] w=\partial^{2} x y[w, \partial z]$. Since $y$ is arbitrary and $R$ is prime, it follows that either $\partial R \subset C$, so (iii) holds by Lemma 3.1 or $\partial^{2} R=0$, a contradiction.

(iii) $\Rightarrow$ (i) is trivial.

THEOREM 3.3. Let $R$ be a prime ring with derivation $\partial$ and $n \in Z^{+}$. If $\partial^{n} x-x \in C$ for all $x \in R$ and if $R$ is $\Delta_{n}$-torsion free then $R$ is commutative.

Proof. Suppose to the contrary that $[x, y] \neq 0$ for some $x, y \in R$. Let $\delta$ be the inner derivation $\delta(z)=[x, z]$ for $z \in R$. Then $\delta$ is not trivial and, by Theorem 2.3, $\delta z \in C$ for all $z \in R$. This contradicts to Lemma 3.1 .

THEOREM 3.4. Suppose $R$ is a prime ring, $\partial$ is a derivation on $R$, and $n>1$ is an even integer. If $\partial^{n} x-x \in C$ for all $x \in R$ and if $R$ is $2 \Delta_{n-1}$-torsion free, then $\partial$ must be trivial.

Proof. Let $x, y \in R . \quad[\partial x, \partial y] \in C$ by Theorem 2.2. Suppose $\partial C=0$. Then $0=\partial[\partial x, \partial y]=\left[\partial^{2} x, \partial y\right]+\left[\partial x, \partial^{2} y\right]$ or $\left[\partial^{2} x, \partial y\right]=$ $-\left[\partial x, \partial^{2} y\right]$. It follows, by repeatedly using the above identity, $[\partial x, \partial y]=\left[\partial^{n} x, \partial y\right]=-\left[\partial^{n-1} x, \partial^{2} y\right]=\left[\partial^{n-2} x, \partial^{3} y\right]=\cdots=-\left[\partial x, \partial^{n} y\right]=$ $-[\partial x, \partial y]$ since $n$ is even. Hence $[\partial x, \partial y]=0$, and by Theorem 3.2 $R$ is commutative. Now suppose $\partial C \neq 0$. Then for $c \in C, \partial c[\partial x, y]=$ $[\partial x, \partial(c y)] \in C$ forces $[\partial x, y] \in C$. Replacing $y$ by $\partial x y$ yields $\partial R \subset C$. Thus, $R$ is commutative by Lemma 3.1.

THEOREM 3.5. Suppose $R$ is a prime ring, $\partial$ is a derivation on $R$, and $n>2$ is an odd integer. If $\partial^{n} x-\partial^{2} x \in C$ for all $x \in R$ and if $R$ is $2(n-2) \Delta_{n-2}$-torsion free, then $\partial$ must be trivial.

Proof. By the corollary to Theorem 2.1, for all $x, y \in R$, $[\partial x, \partial y] \in C$, Suppose $\partial C=0$. Then $\partial[\partial x, \partial y]=0$ yields $\left[\partial^{2} x, \partial y\right]=$ $-\left[\partial x, \partial^{2} y\right]$. On the other hand, by repeatedly using the above identity, we obtain $\left[\partial^{2} x, \partial y\right]=\left[\partial^{n} x, \partial y\right]=-\left[\partial^{n-1} x, \partial^{2} y\right]=\cdots=$ $\left[\partial x, \partial^{n} y\right]=\left[\partial x, \partial^{2} y\right]$ since $n$ is odd. Hence $\left[\partial^{2} x, \partial y\right]=0$. Now, 
replacing $y$ by $\partial y z$ yields $\partial^{2} y\left[\partial^{2} x, z\right]=0$. By replacing $y$ by $\partial y u$ and use the fact $\left[\partial^{2} y, \partial w\right]=0$ for all $w \in R$, we obtain $\partial^{3} y u\left[\partial^{2} x, z\right]=0$. It follows that either $\partial^{3}=0$ or $\partial^{2} x \in C$ for all $x \in R$. If $\partial^{3}=0$ then since $\partial^{n} x-\partial^{2} x \in C, \partial^{2} x \in C$ also. Therefore, by Theorem 3.2, $R$ is commutative. Suppose $\partial C \neq 0$. By using the same argument in the proof of Theorem 3.4 we obtain the commutativity of $R$.

Note that the condition $n$ being odd in Theorem 3.5 is essential and can be seen from the example immediately after Theorem 2.2.

According to Lemma 3.1 and Theorem 3.2, if $R$ is prime of characteristic $\neq 2$ then $\partial^{2} x \in C$ or $\partial x \in C$ for all $x \in R$ implies the commutativity of $R$. A natural question arises whether $\partial^{n} x \in C$ for all $x \in R$, where $n \in Z^{+}$implies the commutativity of $R$. The answer is no in general for $n \geqq 3$. For example, let $R$ be the $2 \times 2$ matrix ring over a division ring of characteristic $\neq 2$ and $a \in R$ with $a \neq 0, a^{2}=0$. Let $\partial$ be the inner derivation $\partial x=[a, x]$ for $x \in R$. Then $\partial^{n} x=a^{n} x-\left(\begin{array}{c}n \\ 1\end{array}\right) a^{n-1} x a+\cdots+(-1)^{n} x a^{n}=0$ for $n \geqq 3$.

We shall show however that if $R$ has no zero divisions $\neq 0$ with certain restriction on its characteristic then $\partial^{n} x \in C$ does imply the commutativity of $R$.

\section{We begin with}

Lemma 3.6. Suppose $R$ is a ring without nilpotent elements $\neq 0$ and suppose $R$ is $(n+1)$-torsion free. If $\partial$ is a derivation on $R$ and $n \in Z^{+}$such that $\partial^{n+1} x=0$ for all $x \in R$ then $\partial^{n} x=0$ for all $x \in R$.

Proof. For $x \in R, 0=\partial^{n+1}\left(x \partial^{n-1} x\right)=(n+1)\left(\partial^{n} x\right)^{2}$ yields $\partial^{n} x=0$.

THEOREM 3.7. Suppose $R$ is a ring without zero divisors $\neq 0$ and $n \in Z^{+}$, and $\partial$ is a nontrivial derivation on $R$. If $\partial^{n} x \in C$ for all $x \in R$ and if the characteristic of $R$ is not a divisor of $(2 n-1)$ !, then $R$ is commutative.

Proof. We proceed by induction on $n$. For $n=1$ or 2 , the commutativity of $R$ follows from Theorem 3.2. Now assume $n>2$. $\partial^{n}\left(\partial^{n-1} x \partial^{n-1} x\right) \in C$ gives $2 \partial^{2 n-1} x \partial^{n-1} x \in C$. By noting that $\partial^{2 n-1} x \in C$, the subgroup argument of Lemma 3.1 shows that either $\partial^{n-1} R \subset C$ or $\partial^{2 n-1} R=0$. In the second case, repeated applications of Lemma 3.6 arrive a contradiction. In the first case $R$ is commutative by induction hypothesis.

We should note that, in Theorem 3.7, if the condition on 
characteristic of $R$ is deleted the ring $R$ need not be commutative. This can be seen from the following example suggested by the referee.

ExAmPLE. Let $R$ be a noncommutative integral domain of characteristic $p>2$ and $x \in R \backslash C$ with $x^{p} \in C$, the center of $R$. Set $\partial(r)=[r, x]$. Especially, let $R$ be the twisted polynomial ring $A[x, T]$ for $A=F[y], F a$ field of characteristic $p$ and $T(f(y))=f(y+1)$ for $f(y) \in A$. Then $\partial^{p} R=0$.

4. Remarks and open problems. In $\S \S 2-3$ if the conditions $\partial^{n} x-\partial^{m} x \in C$ where $m=0,1,2$ and $m<n$ are replaced by $\partial^{n} x+$ $\partial^{m} x \in C$, all results remain true. Moreover, if we relax these conditions by " $\partial^{n} x-\partial^{m} x \in C$ or $\partial^{n} x+\partial^{m} x \in C$ for each $x \in R$ " all results still hold. This can be seen by letting $S=\left\{x \in R \mid \partial^{n} x+\partial^{m} x \in C\right\}$ and $T=\left\{x \in R \mid \partial^{n} x-\partial^{m} x \in C\right\}$ and by noting that $S$ and $T$ are additive subgroups of $R$ with $R=S \cup T$.

For the example following Theorem 3.5, for $n>m \geqq 3$, the condition " $\partial^{n} x \pm \partial^{m} x \in C$ for all $x \in R$ " cannot be expected to imply the commutativity for a prime ring $R$.

We conclude with several open problems.

1. As we pointed out earlier, what are the prime factors of $\Delta_{n}$ ?

2. If, in a ring $R$, for each $x$ there exist positive integers $m$, $n, 0 \leqq m<n$ and $m \leqq 2$, both depend upon $x$, such that $\partial^{n} x-\partial^{m} x \in C$, what can we say about the "commutativity" of $R$ ?

3. In Theorems $2.1,2.2,3.4$ and 3.5, if we assume only that $R$ is 2 -torsion free instead other stronger conditions on the characteristic of $R$, do the results remain true?

4. Does $\partial^{n} x-\partial^{m} x \in C$ for all $x \in R$, where $n>m$, force $\left[\partial^{m} x, \partial^{n} x\right] \in C ?$

5. Suppose, for a ring $R$, there exist a nontrivial derivation $\partial$ and a polynomial $f(t)=a_{n} t^{n}+a_{n-1} t^{n-1}+\cdots+a_{m} t^{m} \in Z[t]$ where $a_{m} \neq 0, \quad a_{n} \neq 0$ and $0 \leqq m \leqq 2$, such that $f(\partial)(x) \in C$ for all $x \in R$, what can we say about the "commutativity" of $R$ ?

Acknowledgment. The authors would like to thank Professors L. Carlitz, R. E. Hartwig, and J. Levine for their interest and many stimulating ideas. They also wish to acknowledge the valuable suggestion given by an anonymous referee in perfecting the final version of this article. Under his (or her) suggestion, Theorems 3.4, 3.5 and 3.7 are improved considerably. Problem 4 is also suggested by the referee. 


\section{REFERENCES}

1. L. Carlitz, A determinant connected with Fermat's last theorem, Proc. Amer. Math. Soc., 10 (1959), 686-690.

2. I. N. Herstein, Noncommutative Rings, John Wiley, 1968.

3. — A note on derivations, Canad. Math. Bull., 21 (1978), 369-370.

4. E. Lehmer, On a resultant connected with Fermat's last theorem, Bull. Amer. Math. Soc., 41 (1935), 864-867.

5. E. C. Posner, Derivations in prime rings, Proc. Amer. Math. Soc., 8 (1957), 10931100 .

Received November 17, 1980 and in revised form May 21, 1981.

North Carolina State University

RALEIGH, NC 27650 


\title{
PACIFIC JOURNAL OF MATHEMATICS
}

\section{EDITORS}

\author{
DONALD BABBITT (Managing Editor) \\ University of California \\ Los Angeles, CA 90024 \\ Hugo RossI \\ University of Utah \\ Salt Lake City, UT 84112 \\ C. C. MOORE and Arthur Agus \\ University of California \\ Berkeley, CA 94720
}

\section{J. DugundjI}

Department of Mathematics

University of Southern California

Los Angeles, CA 90007

R. FinN and J. Milgram

Stanford University

Stanford, CA 94305

\section{ASSOCIATE EDITORS}
R. ARENS
E. F. BeCKENBACH
B. H. NeumanN
F. WOLF
K. YoshidA

\section{SUPPORTING INSTITUTIONS}

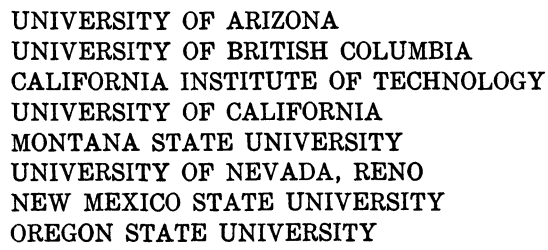

UNIVERSITY OF ARIZONA

UNIVERSITY OF BRITISH COLUMBIA

CALIFORNIA INSTITUTE OF TECHNOLOGY

UNIVERSITY OF CALIFORNIA

MONTANA STATE UNIVERSITY

UNIVERSITY OF NEVADA, RENO

NEW MEXICO STATE UNIVERSITY

OREGON STATE UNIVERSITY

\author{
UNIVERSITY OF OREGON \\ UNIVERSITY OF SOUTHERN CALIFORNIA \\ STANFORD UNIVERSITY \\ UNIVERSITY OF HAWAII \\ UNIVERSITY OF TOKYO \\ UNIVERSITY OF UTAH \\ WASHINGTON STATE UNIVERSITY \\ UNIVERSITY OF WASHINGTON
}

The Supporting Institutions listed above contribute to the cost of publication of this Journal, but they are not owners or publishers and have no responsibility for its content or policies.

Mathematical papers intended for publication in the Pacific Journal of Mathematics should be in typed form or offset-reproduced, (not dittoed), double spaced with large margins. Please do not use built up fractions in the text of the manuscript. However, you may use them in the displayed equations. Underline Greek letters in red, German in green, and script in blue. The first paragraph or two must be capable of being used separately as a synopsis of the entire paper. Please propose a heading for the odd numbered pages of less than 35 characters. Manuscripts, in triplicate, may be sent to any one of the editors. Please classify according to the scheme of Math. Reviews, Index to Vol. 39. Supply name and address of author to whom proofs should be sent. All other communications should be addressed to the managing editor, or Elaine Barth, University of California, Los Angeles, California, 90024.

50 reprints to each author are provided free for each article, only if page charges have been substantially paid. Additional copies may be obtained at cost in multiples of 50 .

The Pacific Journal of Mathematics is issued monthly as of January 1966. Regular subscription rate: $\$ 102.00$ a year (6 Vols., 12 issues). Special rate: $\$ 51.00$ a year to individual members of supporting institutions.

Subscriptions, orders for numbers issued in the last three calendar years, and changes of address shoud be sent to Pacific Journal of Mathematics, P.O. Box 969, Carmel Valley, CA 93924, U.S.A. Old back numbers obtainable from Kraus Periodicals Co., Route 100, Millwood, NY 10546.

\section{PUBLISHED BY PACIFIC JOURNAL OF MATHEMATICS, A NON-PROFIT CORPORATION}

Printed at Kokusai Bunken Insatsusha (International Academic Printing Co., Ltd.). 8-8, 3-chome, Takadanobaba, Shinjuku-ku, Tokyo 160, Japan. 


\section{Pacific Journal of Mathematics}

Vol. 99, No. $2 \quad$ June, 1982

Thomas E. Armstrong and Karel Libor Prikry, On the semimetric on a

Boolean algebra induced by a finitely additive probability measure . . . .249

Walter Russell Bloom, Strict local inclusion results between spaces of

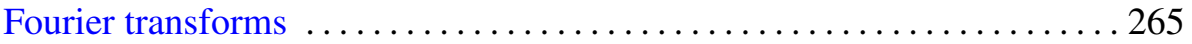

Richard Clark Brown, Notes on generalized boundary value problems in

Banach spaces. II. Infinite-dimensional extension theory ........... 271

Sui Sun Cheng, Isoperimetric eigenvalue problem of even order differential

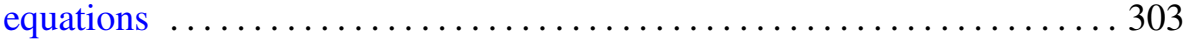

Lung O. Chung and Jiang Luh, Derivations of higher order and

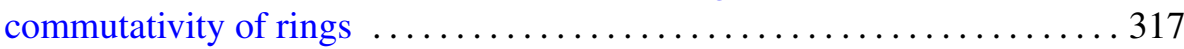

Ali Ahmad Fora, A fixed point theorem for product spaces . . . . . . . . . 327

Barry J. Gardner, Radical classes of regular rings with Artinian primitive

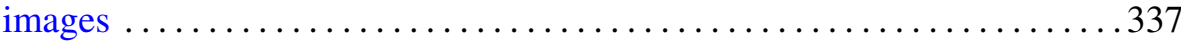

John Brady Garnett and Peter Wilcox Jones, BMO from dyadic BMO . . . 351

Allen E. Hatcher, On the boundary curves of incompressible surfaces . . . . 373

Richard Howard Hudson and Kenneth S. Williams, Resolution of

ambiguities in the evaluation of cubic and quartic Jacobsthal sums .....379

Viktor Losert, Counter-examples to some conjectures about doubly

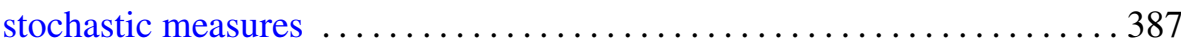

Kenneth Derwood Magill, Jr., P. R. Misra and Udai Bhan Tewari,

Structure spaces for sandwich semigroups

Mark Mandelker, Continuity of monotone functions

Kenneth Guy Miller, An index theorem and hypoellipticity on nilpotent Lie groups ......................................... 419

Evelyn M. Nelson, Homomorphisms of mono-unary algebras . . . . . . . . . 427

Marvin E. Ortel, The support of an extremal dilatation . . .

R. S. Pathak and O. P. Singh, Finite Hankel transforms of distributions . . . 439

Richard Cole Penney, The theory of ad-associative Lie algebras

Linda Ruth Sons, Zero distribution of functions with slow or moderate

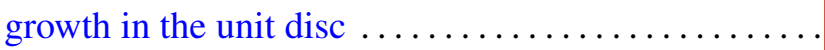

Russell Bruce Walker, Transversals to laminations 